\title{
Protótipo digital do Cuidando Bem: um jogo educacional sobre Segurança do Paciente
}

\author{
Aline Natalia Domingues ${ }^{1}$, Ana Esther Camargo ${ }^{2}$, Jessica Sayuri Nishiguchi², \\ Marcelo Lopes Lotufo ${ }^{2}$, Rogério Augusto Bordini², Luiz Valério Neto ${ }^{2}$, Delano \\ Medeiros Beder ${ }^{2}$, Joice Lee Otsuka², Sílvia Helena Zem-Mascarenhas ${ }^{1}$ \\ ${ }^{1}$ Departamento de Enfermagem, Universidade Federal de São Carlos- Brasil \\ ${ }^{2}$ Laboratório de Objetos de Aprendizagem, Universidade Federal de São Carlos- Brasil \\ aline.natalia@sead.ufscar.br, \{annaerodrigues, marlolo1993,
rogerbordini, luizvneto, silviazem\}@gmail.com,
jessica_sayuril@hotmail.com, \{delano, joice\}@dc.ufscar.br
}

Abstract. This paper presents the educational game Taking Good Care, that aims to teach patient safety protocols for students of professional courses in nursing. This article presents the main functionalities of this game, its development, emphasing the results obtained by testing the first digital prototype of this game. It is believed that the results of this study should provide evidence to support the importance of innovative strategies in health, as well as to encourage the development of other educational games for Health.

Resumo. O presente artigo apresenta o jogo educativo Cuidando Bem, que tem como objetivo ensinar os protocolos de segurança do paciente para estudantes de cursos profissionalizantes em enfermagem. Neste artigo são apresentados as principais características do jogo, o seu desenvolvimento, enfatizando os resultados obtidos nos testes realizados com os primeiros protótipos digitais do jogo. Espera-se que os resultados deste estudo forneçam evidências que sustentem a importância de estratégias inovadoras na área da saúde, bem como incentive o desenvolvimento de outros jogos educacionais para a Saúde.

\section{Introdução}

A assistência segura é um tema de relevância no contexto assistencial, tornando-se um assunto prioritário na área de saúde. A garantia de um cuidado seguro e livre de iatrogenias envolvem esforços de todo um sistema, que deve ser iniciado desde o processo de formação educacional dos profissionais. O Programa Nacional de Segurança do Paciente traz como estratégia de implementação a articulação com o Ministério da Educação e o Conselho Nacional de Educação, para inclusão do tema segurança do paciente nos currículos dos cursos de formação em saúde de nível técnico, superior e pós-graduação na área da saúde [Brasil 2013]. Portanto, considerando o lançamento do Programa Nacional de Segurança do Paciente pelo Ministério da Saúde em 2013 e a importância deste tema, as instituições dos cursos profissionalizantes de nível médio em enfermagem devem se preocupar em relação à reflexão sobre a segurança do paciente na aprendizagem de seus alunos.

O avanço tecnológico dos últimos anos está propiciando a melhoria da prestação de serviço em diversas áreas e, no contexto educacional, novos aplicativos e equipamentos estão sendo utilizados, favorecendo os processos educacionais com o uso de ambientes realistas e interativos. A abordagem educacional baseada em jogos educacionais podem integrar características lúdicas a conteúdos específicos, motivando 


\section{CBIE-LACLO 2015}

Anais dos Workshops do IV Congresso Brasileiro de Informática na Educação (CBIE 2015)

o processo de aprendizado [Machado et al. 2011]. A aprendizagem baseada em jogos permite que os estudantes desenvolvam atitudes e aptidões, como também, interatividade [Monsalve 2014], [Oblinger 2004]. Para Squire (2007) o uso de jogos no processo-ensino-aprendizagem propicia não somente o entendimento teórico da experiência usando a tecnologia, mas também obtém diretrizes e vivências práticas. Jogos permitem aprendizado através de falhas, desenvolvimento de identidade através do entendimento e de resolução de problemas [Monsalve 2014].

É nesse contexto que o Laboratório de Objetos de Aprendizagem (LOA) da UFSCar vem desenvolvendo suas pesquisas concentradas na utilização dos games como objetos capazes de proporcionar um aprendizado lúdico ao estudante/jogador. Um dos projetos em desenvolvimento é o Cuidando Bem, um jogo do gênero point and click, que tem como objetivo educacional criar situações que promovam a compreensão dos protocolos de segurança dos pacientes, estabelecidos pelo Ministério da Saúde [Brasil, 2013], por meio da análise e reflexão sobre a sua importância e uso adequado. O jogo Cuidando Bem tem como público-alvo primário estudantes de cursos técnicos em Enfermagem, mas deverá ser acessível a todas as pessoas que queiram conhecer e compreender os protocolos de segurança, dada a importância do tema.

Este estudo apresenta o processo de design do Cuidando Bem, com ênfase na realização de testes do game com o público-alvo, como uma prática fundamental na avaliação de protótipos, para a verificação da qualidade dos elementos de jogabilidade e conteúdo educacional. Desse modo, esse trabalho está dividido em diferentes seções, assim como relatadas a seguir: a seção 2 descreve a metodologia de pesquisa utilizada neste trabalho. Na seção 3 é apresentada a fundamentação teórica do trabalho na seção 4 alguns trabalhos relacionados. A seção 5 apresenta o processo de design do jogo Cuidando Bem, na seção 6 apresentado o protótipo e seção 7 análise dos resultados. Finalmente, na seção 8 são apresentadas as considerações finais do trabalho.

\section{Metodologia do trabalho}

Os seguintes procedimentos metodológicos estão sendo desenvolvidos ao longo do projeto, não necessariamente nesta sequência:

1. Estudos e pesquisas nas áreas de design e avaliação de jogos educacionais, bem como sobre o conteúdo específico e trabalhos relacionados;

2. Levantamento de requisitos sobre o conteúdo específico com professores do Ensino Técnico e de graduação em Enfermagem;

3. Concepção do jogo (brainstorms, design de níveis com ênfase na tétrade elementar: mecânica, narrativa, estética e tecnologia) com base em demandas identificadas e criação de um Game Design Document (GDD);

4. Desenvolvimento de componentes audiovisuais, codificação e testes internos, que vêm sendo desenvolvidos em ciclos iterativos;

5. Elaboração de protótipos em papel e digital, com o intuito de validar as soluções de design propostas;

6. Testes internos e com o público-alvo (professores e estudantes do Ensino Técnico e Graduação), ao longo do desenvolvimento do jogo.

O desenvolvimento do projeto está sendo realizado por estudantes de graduação e pós-graduação (Computação, Enfermagem, Imagem e Som, Letras e Música). O projeto tem contado com a colaboração de professores de Enfermagem do ensino técnico e superior, na revisão dos conceitos educacionais presentes no projeto. Para tanto, foram 


\section{CBIE-LACLO 2015}

Anais dos Workshops do IV Congresso Brasileiro de Informática na Educação (CBIE 2015)

disponibilizados aos professores um espaço virtual e um questionário on-line, por meio dos quais os professores têm contribuído com dúvidas, críticas e sugestões. Esses espaços têm sido importantes também para a avaliação de questões específicas referentes à utilização do game enquanto recurso didático.

\section{Fundamentação teórica}

Prensky (2012) observa a necessidade de se rever as teorias e práticas educacionais para que estejam mais adequadas a novos estilos de aprendizagem, e defende que essas práticas sejam baseadas em jogos digitais, justamente por estarem alinhadas com as necessidades e os estilos de aprendizagem da geração atual e futura; por motivar pela diversão; e por ser versátil, podendo ser adaptada a diferentes disciplinas, informações ou habilidades a serem aprendidas. A aprendizagem baseada em jogos (Games-Based Learning) é defendida por Squire (2007) como uma forma de engajar alunos em atividades significativas e permitir que assumam novas identidades, explorem mundos e aprendam. Segundo Gee (2005), os bons jogos trazem bons princípios de aprendizagem, caso contrário os jogadores não aprenderiam a jogá-los e perderiam o interesse por eles. Gee (2005) analisa os princípios de aprendizagem dos bons jogos e como esses princípios podem ser explorados em um contexto educacional.

Os jogos educacionais são feitos para divertir e aumentar a chance da aprendizagem de conceitos, conteúdos e habilidades embutidos no jogo, proporcionando ao aluno um ambiente de aprendizagem rico e complexo [Azzolini, Assis e Pontarolo 2014]. Alguns autores denominam esses jogos de "micromundos, nos quais se aprende [Silva, Bernardi e Müller 2012]. Para Prensky (2012) o que se pretende hoje é a aprendizagem baseada em jogos digitais de modo que o jogo assuma o papel central em promover a aprendizagem do aluno.

Para o processo de game design, foram utilizados a metodologia de Novak (2010) e Schell (2011). Novak (2010) traz o desenvolvimento de um jogo a partir de cinco etapas: conceito, pré-produção, protótipo, produção e pós produção. Já no escopo da literatura de game design, destacamos o trabalho de Schell (2011) que define um jogo como composto por quatro elementos que compõem o que ele denominou a tétrade elementar (estética, mecânica, narrativa e tecnologia).

As novas tecnologias estão sendo cada vez mais utilizadas e integradas nos currículos da saúde, permitindo aos alunos o desenvolvimento de novas habilidades dentro de um ambiente seguro [Dariel et al. 2013]. A inserção de novos recursos no contexto educacional, entre eles o serious game, como um potente instrumento pedagógico. Estes jogos, com propósitos e conteúdos específicos, são chamados serious game (jogos sérios) e permitem apresentar situações novas, discutir melhores formas de resolução, além de possibilitar a construção de conhecimentos e treinamento de atividades particulares [Blackman 2005], [Zyda 2005], [Machado et al. 2011]. O serious game é caracterizado como um jogo educacional que promove a construção do conhecimento através de uma proposta pedagógica com conteúdos específicos, aliado a aspectos lúdicos que estimulam e motivam os processos de ensino aprendizagem [Abt 1987], [Fuchslocher, Niesenhaus, Krämer 2011].

O que difere o serious game dos demais tipos de jogos é o foco em resultados específicos de aprendizagem e também para alcançar mudanças de desempenho e de comportamentos que podem ser mensuráveis e continuadas [Machado et al. 2011]. Nesta perspectiva o jogo educacional Cuidando Bem, pode ser caracterizado como um serious game. 


\section{Trabalhos relacionados}

Para a formulação tanto do conteúdo pedagógico quanto da estruturação da mecânica, foi realizada uma pesquisa de jogos que apresentam ideias semelhantes às do jogo proposto neste artigo.

“e-Baby" é um jogo educativo no estilo point-click desenvolvido numa parceria entre a Escola de Enfermagem de Ribeirão Preto da Universidade de São Paulo - Brasil e Escola Superior de Enfermagem de Coimbra - Portugal. Nele, o usuário é apresentado a um caso clínico, no qual deverá oferecer suporte a um recém-nascido pré-termo [GPECCA 2014].

"Digestower" é um jogo educativo no estilo tower defense, voltado para o público de estudantes do ensino técnico de enfermagem e crianças, também desenvolvido no LOA, com a finalidade de explorar alguns conceitos de anatomia e fisiologia do sistema digestório humano, além de outros aspectos, como os malefícios da gordura no organismo, o efeito das vitaminas e do exercício físico [Dias et al. 2014].

Observou-se também que os jogos acima descritos possuem objetivo educativo e foram elaborados com o intuito de colaborar na formação do processo ensinoaprendizagem de alunos ou profissionais da área da saúde, aspecto semelhante ao do Cuidando Bem.

\section{O jogo Cuidando Bem}

O desenvolvimento do jogo está ocorrendo em etapas. Nas subseções seguintes são apresentadas breves considerações, sobre cada etapa no projeto em questão, para maior detalhamento, os resultados desta seção ver Domingues et al. (2014a).

\subsection{Levantamento de requisitos do jogo}

O Cuidando Bem tem como objetivo primordial o desenvolvimento de habilidades e competências no que tange a aprendizagem sobre segurança do paciente, com o intuito de ensinar aos alunos do curso técnico em enfermagem, os protocolos lançados pelo Ministério da Saúde.

\subsection{Concepção}

A partir dos requisitos delimitados para o projeto, a equipe iniciou o processo de concepção do jogo, tendo como base de design a Tétrade Elementar proposta por Schell (2011), na qual foi possível conceber os primeiros modelos de estética, mecânica, narrativa e tecnologia a serem utilizados. Os principais resultados desta etapa serão apresentados resumidamente, a seguir. Para maior detalhamento desta etapa, verificar o trabalho Domingues et al. (2014b).

- Narrativa: O jogo trata os primeiros três meses de experiência de um técnico de enfermagem em um hospital. Os casos clínicos serão distribuídos em nove fases, equilibrando o nível de dificuldade do jogo.

- Mecânica: A mecânica do jogo se baseará no controle do protagonista que é feito através de interações no cenário utilizando apenas o mouse (point-click). Utilizando um sistema point and click o jogador deverá utilizar os objetos no cenário, ações e interações com outros personagens para avançar nas fases.

- Estética: O estilo visual escolhido para o desenvolvimento das ilustrações perpassa o uso de traços estilizados para um jogo em 2D, com animações simples e equilíbrio assimétrico. 
CBIE-LACLO 2015

Anais dos Workshops do IV Congresso Brasileiro de Informática na Educação (CBIE 2015)

- Tecnologia: Optou-se pelo uso do HTML 5 (Hypertext Markup Language, versão 5) por suportar múltiplas plataformas de hardware e software, considerando que é uma linguagem multiplataforma e a única exigência para sua execução é a presença de navegadores que estejam em conformidade com a especificação.

\section{Protótipo}

A partir da concepção de design do jogo descrita na seção anterior, foi criado o protótipo para a validação dos requisitos apresentados na subseção 5. 2.

De acordo com Rogers et al. (2013), "um protótipo é uma manifestação de um design que permite aos stakeholders interagirem com ele e explorarem sua adequação; ele é limitado na medida em que um protótipo normalmente enfatiza um conjunto de características do produto e não outras". Dessa forma, protótipos respondem a questões e fornecem suporte aos designers para a escolha de uma dentre as várias opções. Portanto, protótipos servem para vários fins: testar a viabilidade técnica de uma ideia, esclarecer alguns requisitos vagos, realizar testes e avaliações com usuários ou verificar se certo rumo que se tomou ao projeto é compatível com o resto do desenvolvimento do sistema [Santa Rosa e Moraes 2012]. Para estes autores, os protótipos podem ser classificados em duas categorias [Rogers et al. 2013], [Santa Rosa e Moraes 2012], o protótipo de baixa fidelidade que é aquele que não se parece muito com o produto final e o protótipo de alta fidelidade utiliza materiais que se espera que estejam no produto final e, portanto, se assemelha em muito ao resultado final pretendido.

Dessa forma, no projeto Cuidando Bem foram desenvolvidos protótipos em papel nas fases iniciais de design, com o intuito de validar, de forma rápida e sem muito custo, a mecânica básica do jogo. Foram realizadas sessões com integrantes do grupo, pessoas externas ao grupo e também com uma pessoa cega (o jogo deverá ser acessível para pessoas com deficiência visual). $\mathrm{O}$ resultado dos testes com os protótipos em papel foram apresentados em Domingues et al. (2014c).

Após a prototipação em papel, foi desenvolvido um protótipo digital de alta fidelidade em HTML 5, contemplando a mecânica básica, parte da narrativa e já com as principais ilustrações elaboradas para o jogo. Esse protótipo também contemplou o design da interface do jogo, possibilitando testes iniciais de usabilidade e jogabilidade.

Já o protótipo digital de alta fidelidade apresentado neste trabalho, possui características mais próximas ao desenvolvimento esperado da versão final do jogo. Nesta versão são apresentados apenas a fase introdutória do jogo e a primeira fase, consistindo em dois casos clínicos de nível fácil de compreensão, para que o jogador se ambiente com a interface e jobabilidade.

A interação com o jogo ocorre através do mouse (point-click). Se quiser se movimentar para outro cenário, o jogador poderá clicar no caminho correspondente a esse lugar que deseja ir, os diálogos também, vão para as próximas falas clicando nas setas dos balões. Além disso, é possível interagir com os objetos clicando sobre eles, ou clicando e arrastando o objeto, para interagir o objeto selecionado com outro elemento do cenário, como exemplo o caso de precisar aferir a temperatura no paciente. Abaixo são exibidas algumas telas destas fases do jogo, Figura 1 apresenta o leito do paciente e o cenário do posto de enfermagem, com os objetos de interação na tela. 


\section{CBIE-LACLO 2015}

Anais dos Workshops do IV Congresso Brasileiro de Informática na Educação (CBIE 2015)

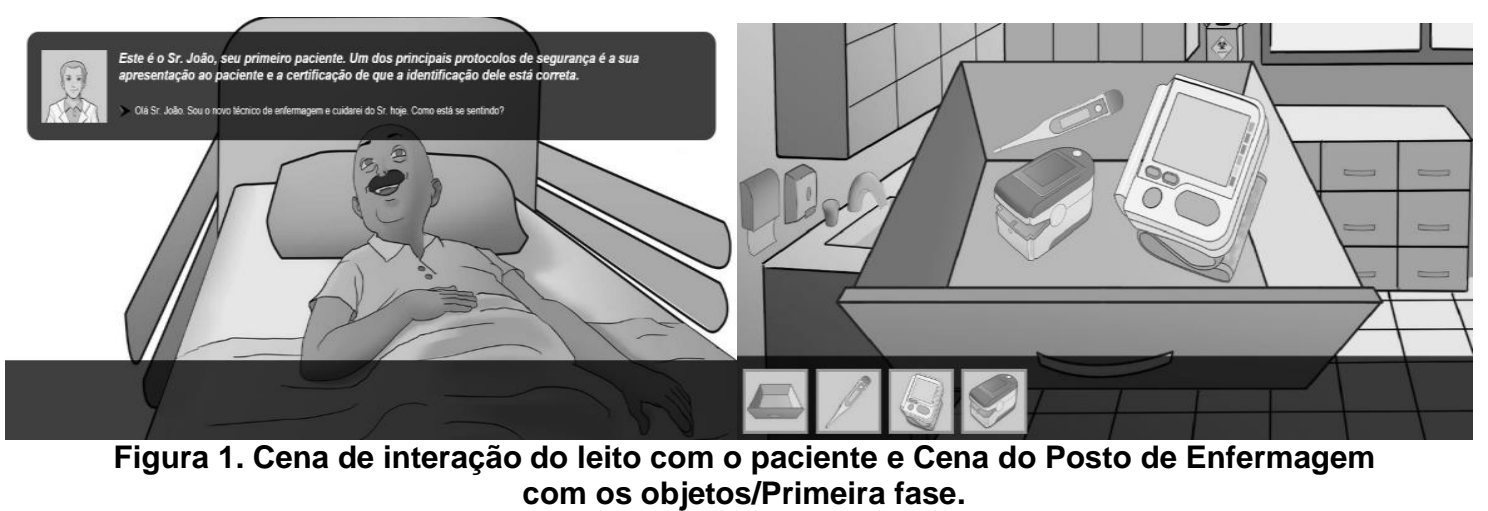

Com este primeiro protótipo digital foi possível a realização dos primeiros testes com pessoas do público-alvo, ou seja, estudantes do Ensino Técnico em Enfermagem. Na próxima seção são apresentados os principais resultados obtidos. A avaliação testada do jogo Cuidando Bem é caracterizado como um protótipo digital de alta fidelidade.

\subsection{Primeiros testes com o protótipo digital}

Nesta seção são apresentadas a avaliação do Cuidando Bem. Para a garantia dos direitos dos participantes foram respeitados todos os aspectos contidos na resolução 466/12 do Conselho Nacional do Ministério da Saúde que dispõe sobre as diretrizes para a realização de pesquisas com seres humanos [Brasil 2012]. O projeto de pesquisa foi aprovado pelo Comitê de Ética em Pesquisa da Universidade Federal de São Carlos sob o parecer de número 750.184 e todos os participantes assinaram o Termo de Consentimento Livre e Esclarecido. A seção de teste do jogo foi realizada no mês de novembro de 2014, em uma escola estadual de ensino técnico de um município do interior do estado de São Paulo, no escopo de uma disciplina denominada como Fundamentos de Enfermagem I, no período matutino, devido que estes alunos se encontravam no primeiro módulo do curso, já que o mesmo é dividido em quatro módulos em um período de dois anos. Esta escolha ocorreu devido ao fato que os pesquisadores optaram por apresentar o jogo inicialmente para alunos, que estão iniciando sua aprendizagem na área de enfermagem, sobre a perspectiva que a segurança do paciente deve ser introduzida desde o primeiro contato dos alunos com o curso. A análise dos dados foram realizadas por meio de estatística simples.

Participaram da realização do teste de avaliação do protótipo digital um total de dezoito alunos. A faixa etária variou entre de 18 ao 59 anos, com uma média de idade de 27 anos. Em relação à escolaridade dos alunos apenas um possuía Ensino Médio Incompleto, pois ainda estava cursando e um aluno possuía Nível Superior Incompleto, os outros dezesseis alunos, possuíam Ensino Médio Completo. Em relação a questão de gênero, a predominância da composição da amostra foi feminina com $82 \%$. Além disso, foram analisados a preferência do uso de dispositivos para jogos, evidenciando que $46,9 \%$, tinham preferência pelo uso simultâneo de celular e smartphones/notebook e computador para jogar. 
CBIE-LACLO 2015

Anais dos Workshops do IV Congresso Brasileiro de Informática na Educação (CBIE 2015)

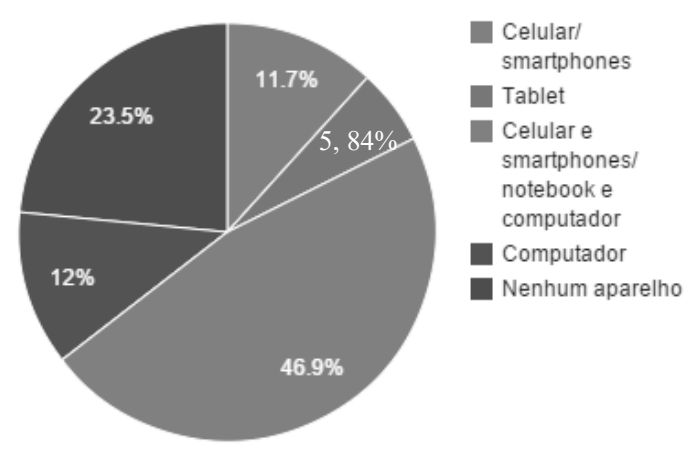

Figura 2. Distribuição da preferência do uso de dispositivos para jogos. São Carlos, SP, 2014.

A sessão de teste, ocorreu da seguinte forma, primeiramente foram apresentados o escopo do projeto de pesquisa, os objetivos do teste e a dinâmica da sessão. Posteriormente foi solicitado aos alunos que jogassem o jogo educativo em um dos computadores do laboratório de informática da escola. Durante o momento que jogavam um dos designers observava a interação dos mesmos com o jogo, sem realizar nenhuma intervenção direta ao testador/alunos. Após o término do jogo, foi entregue um questionário misto para que os alunos respondessem algumas questões pertinentes a concepção do jogo. Este instrumento elaborado teve predominância de questões fechadas, com três opções de seleções (sim, não, qual (s)) e uma questão aberta de sugestão para melhoria do jogo. Abaixo segue algumas das questões do instrumento elaborado para a avaliação do protótipo:

- Está claro no jogo os objetivos e suas regras?

- O jogo precisa melhorar o feedback? Em relação ao jogo Cuidando Bem você encontrou alguma dificuldade de navegação?

- Você gostaria de utilizar este jogo para aprender sobre a temática de segurança do paciente?

- Você acredita que este jogo possa ser utilizado como um recurso novo de aprendizagem para o ensino profissionalizante de enfermagem?

- Em relação aos aspectos da interface do jogo apresentado (ilustrações, cenários, fases iniciais do jogo), você acredita que está adequado?

- Você tem alguma sugestão para melhoria da proposta do jogo Cuidando Bem?

- Você tem interesse de participar da sessão de testes das próximas fases?

Além das questões apresentadas acima, foi concedido espaço aberto informal, para que os alunos pudessem se expressar livremente, tanto em relação aos elementos do jogo que mais lhes chamaram a atenção, quanto àqueles que necessitam ser revisados.

Todos os participantes (100\%) responderam que estavam claros o objetivo do jogo, não encontrando nenhuma discordância do que foi apresentado. A avaliação dos alunos em relação aos aspectos da interface do jogo, referente às ilustrações, cenários, fases inicias do jogo se estão adequados de acordo com a sua percepção de compreensão, todos os participantes (100\%) responderam que está adequado. Cerca de $88 \%$ afirmaram que utilizariam este jogo para aprender sobre a temática de segurança do paciente. Foi avaliado também o feedback do jogo em relação às ações do jogador, elemento essencial para que o jogador/aprendiz perceba se os efeitos de suas ações no mundo do jogo e se está avançando em relação aos seus objetivos. A concordância dos 
CBIE-LACLO 2015

Anais dos Workshops do IV Congresso Brasileiro de Informática na Educação (CBIE 2015)

alunos frente ao feedback do jogo foram de $82,4 \%$ responderam que sim e apenas $17,6 \%$, responderam que não.

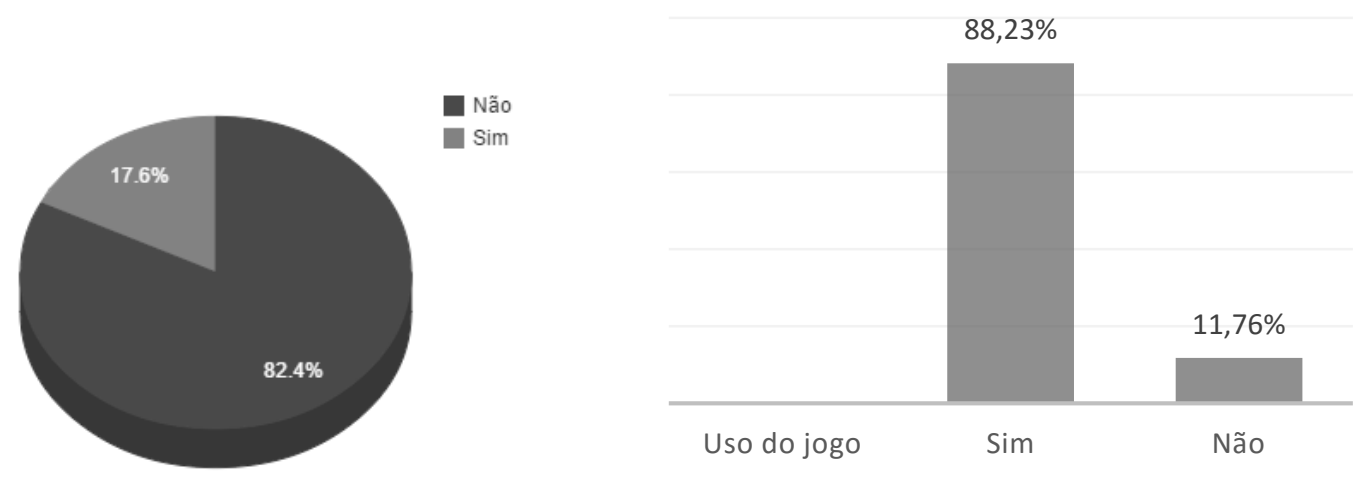

Figura 3. Percepção dos alunos sobre o feedback do jogo. São Carlos, SP, 2014.

Figura 4. Utilização do jogo para o ensino. São Carlos, SP, 2014.

Os participantes também opinaram sobre sugestões de melhoria para o jogo, dentre as questões apresentadas estavam voltadas ao retorno de melhoria do feedback ao jogador, por exemplo, após realizar uma ação de verificação de identificação do paciente pela pulseira, que tivesse algo no jogo, mostrando que essa ação já havia sido realizada. Outra aspecto que relataram foi a possibilidade de que o jogador possa incluir anotações no prontuário do paciente. Além disso, todos os participantes responderam que têm o interesse em avaliar futuramente novas versões do jogo. Por outro lado, os testes apontaram que o jogo contém algumas falhas relacionadas principalmente à narrativa e à jogabilidade, cujas indicações e sugestões dos alunos e designers da equipe são relatadas a seguir:

- Colocar mais opções de respostas no diálogo do jogador, para que o mesmo tenha mais opções dentro do jogo e possibilitar aleatoriedade nas respostas para que o jogador não decore a disposição do texto dentro dos balões e mecanize a suas ações dentro do jogo.

- Alterar mecânica de verificação das ações realizadas pelo jogador, oferecer feedback ao jogador; adicionar ao fim de cada fase a porcentagem de acertos das ações realizados pelo jogador e inserir anotações no prontuário do paciente, referente as ações realizadas com os pacientes nas fases do jogo.

\section{Análise dos resultados}

Os feedbacks apresentados pelos alunos por meio dos testes de refinamento do jogo, foram de grande importância para que a equipe identificasse aspectos que requerem especial atenção, com o intuito de promover melhorias e oferecer uma experiência lúdica e educacional correspondente às expectativas do público-alvo, visto que alguns problemas relatados pelos jogadores, foram anteriormente apontados pelos próprios desenvolvedores e designers, enfatizando a importância da realização dos testes, no desenvolvimento do jogo educacional. A realização do teste do protótipo permite identificar a maior quantidade de falhas e requisições de mudanças, evitando que tais informações sejam levantadas próximas ao final do projeto, quando se tem pouco tempo e recurso restante [Fernandes e Dias 2006]. Através do teste de avaliação do protótipo 
do Cuidando Bem foi possível analisar a opinião do público-alvo em relação ao recurso educacional contribuindo de forma significativa com sugestões de melhoria.

Ao final do processo de testes, pode-se concluir que o Cuidando Bem está percorrendo um caminho de desenvolvimento adequado e satisfatório na perspectiva de atingir seus requisitos e os objetivos educacionais. Consideramos que todos os resultados obtidos até momento são satisfatórios e contribuíram para medir e melhorar a qualidade do jogo.

\section{Considerações finais}

O presente estudo teve como intuito apresentar o primeiro protótipo digital do jogo Cuidando Bem, um jogo educativo sobre segurança do paciente e os resultados principais dos primeiros testes realizados com esse protótipo.

Podemos concluir que a prototipação de alta fidelidade contribuiu de forma significativa, pois proporcionou uma maior avaliação das decisões do projeto a serem tomadas pela equipe responsável pelo game design, além de ressaltar a importância do design participativo desde as fases iniciais do desenvolvimento de um jogo. Este design participativo consiste no trabalho de vários profissionais da área da Saúde e da Computação, além da interação fundamental com o próprio público-alvo e os docentes que utilizarão o recurso, com o intuito de construir um jogo alinhado com as necessidades dos docentes e expectativas dos estudantes, ou seja, com conteúdos e mecânicas apropriados para se alcançar os objetivos educacionais pretendidos, de forma lúdica.

O uso de novas tecnologias no processo de aprendizagem dos alunos permite que os mesmos desenvolvam habilidades, dentro de um ambiente seguro. Desta forma, o uso de jogos educativos no processo educacional possibilita a apresentação de novas situações clínicas e construção de novos conhecimentos, através de um ambiente diferenciado lúdico e inovador, que estimula o processo de ensino-aprendizagem.

Acredita-se que o jogo Cuidando Bem possibilitará a sua utilização no processo de ensino e aprendizagem no contexto da segurança do paciente. O jogo ainda está em fase de desenvolvimento e novos testes serão realizados. Após a finalização, será possível a realização de uma avaliação mais profunda sobre o nível de impacto do uso do jogo na área da Enfermagem. Espera-se que os resultados deste estudo forneçam evidências que sustentem a importância de estratégias inovadoras na área da saúde, bem como incentive o desenvolvimento de outros jogos educacionais para a Saúde.

\section{Referências}

ABT, C.C. (1987), Serious Games. New York, NY: Viking Press, 177p.

Azzolini, V.L., Assis, G.A, Pontarolo, E. (2014), CidTrans: Um Jogo para Educação de Condutores no Trânsito Urbano. XXV Simpósio Brasileiro de Informática na Educação (SBIE 2014).

Blackman, S. (2005). Serious Gamens... and Less! Computer Graphics, 39 (1): 1216. ACM.

Brasil, Ministério da Saúde (2013), Portaria n.529, de 1. ${ }^{\circ}$ de abril de 2013. Diário Oficial da República Federativa do Brasil, Brasília (DF).

Brasil, Ministério da Saúde (2012), Diretrizes e normas regulamentadoras de pesquisa envolvendo seres humanos, Brasília (DF). 


\section{CBIE-LACLO 2015}

Anais dos Workshops do IV Congresso Brasileiro de Informática na Educação (CBIE 2015)

Dariel, O.J.P. et at. (2013). Developing the Serious Games potential in nursing education. Nurse Education Today, v.33, p. 1569-1575.

Dias, J.D. et al. (2014). DIGESTOWER: jogo educacional para auxiliar o enfrentamento da obesidade infantil.In: XIX Conferência Internacional sobre Informática na Educação (TISE2014).

Domingues, A.N. et al. (2014a). Domingues, A.N. et al. (2014). Cuidando Bem: um serious game sobre segurança do paciente. XIV Congresso Brasileiro de Informática em Saúde (CBIS 2014).

Domingues, A.N. et al. (2014b). Desenvolvimento de Jogo Educativo sobre Segurança do Paciente para Ensino Profissionalizante de Enfermagem. In: II Simpósio Internacional de Educação a Distância (SIED:ENPED 2014). FullPaper.

Domingues, A.N. et al. (2014c). Uso de protótipo em papel no design de um jogo educacional acessível. In: XIII Simpósio Brasileiro de Jogos e Entretenimento Digital (SBGames 2014).

Fernandes, T. e Dias, A. (2006) Processo de Testes para Desenvolvimento de Jogos Celulares. In Procedings of the V Brazilan Symposium on Computer Games and Digital Enterainment (SBGames Computing). ShortPaper.

Fuchslocher, A., Niesenhaus, J., Krämer, N. (2011), Serious games for health: An empirical study of the game "Balance" for teenagers with diabetes mellitus. Entertainment Computing, v.2, p. 97-101.

Gee, J. P. (2005). Good video games and good learning. Phi Kappa Phi Forum, no 2.

GPECCA. (2014). Grupo de pesquisa em enfermagem no cuidado à criança e ao adolescente. Escola de Enfermagem de Ribeirão Preto-USP.

Machado, L. dos S. et al., (2011). Serious Games Baseados em Realidade Virtual para Educação Médica. Revista Brasileira de Educação Médica, n.35, v.2, p. 254262.

Monsalve, E.S. (2014) "Uma abordagem para Transparência Pedagógica usando Aprendizagem Baseada em Jogos", Pontifícia Universidade Católica do Rio de Janeiro.

Novak, J. (2010). Game development essentials: an introduction. Ed. Cengage Learning.

Oblinger, D. (2004). The Next Generation of Educational Engagement. Journal of Interactive Media in Education.

Prensky, M. (2012) “Aprendizagem baseada em jogos digitais”. São Paulo: Editora Senac São Paulo.

Rogers, Y.; Sharp, H. \& Preece, J. (2013). "Design de Interação: Além da Interação Humano-Computador. 3. Ed. Porto Alegre, Brasil: Bookman.

Santa Rosa, J.G, Moraes, A. (2012), Avaliação e projeto no design de interfaces. Teresópolis, RJ: 2AB.

Schell, J (2011). "The Art of Game Design: A Book of Lenses". Morgan Kaufman: Mellon University.

Silva, T.C., Bernardi, G., Müller, F.M. (2012), Abordagem de Apoio ao Ensino e Aprendizagem de Teste de Software Baseada em Jogos Sérios e Mundos Virtuais. XXII Simpósio Brasileiro de Informática na Educação (SBIE- XVII WIE 2012).

Squire, K. D. (2007) “Games, Learning and Society: Building a Field”. Educational Technology. p. 52-53.

Zyda, M. (2005), From Visual Simulation to Virtual Reality to Games. In: Computer. IEEE Computer Society, v.38, n.9, p. 2532. 